\title{
Positioning and Orienting a Class of Symmetric Parts Using a Combination of a Unit-Radial and a Constant Force Fields
}

\author{
Florent Lamiraux Lydia E. Kavraki \\ Department of Computer Science \\ Rice University, Houston, TX, USA \\ \{lamiraux, kavraki\}ecs.rice.edu
}

\begin{abstract}
Part positioning and orientation is a key issue in manufacturing. Recent approaches perform this task using force fields over a plane. Force fields can be implemented in the microscale with MEMS actuator arrays and in the macroscale with mechanical motors or small air jets. A part placed on a force field is subjected to a resultant force and torque acting on the contact surface of the part. As a result, the part moves toward an equilibrium configuration. Extensive recent work has investigated a series of force fields for part positioning and orientation. Typically, a strategy that brings a part to a unique equilibrium consists of several force fields that are employed in sequence. Böhringer and Donald conjectured a few years ago that the combination of a unit radial field with a constant field would give rise to a unique equilibrium. Such a field is extremely interesting as it positions and orients parts without the need of sensing or a clock. Recently we proved this conjecture for non symmetric parts. In this paper, we focus our attention on symmetric parts and we show that some of them can be uniquely positioned and oriented using the same field. Our work further explores the capabilities and limits of force fields and provides additional evidence that force fields are a powerful tool for parts manipulation.
\end{abstract}

\section{Introduction}

During manufacturing, parts typically stored in boxes have to be manipulated and oriented before assembly. This task is critical in manufacturing since it strongly affects the productivity of the assembly line. Orientation has been traditionally performed by vibratory bowl feeders. However, these devices are usually designed for the orientation of a single part and need to be redesigned if the shape of the part changes.

Recent work has investigated alternative ways of orienting parts in assembly workcells. Programmable part feeders are more flexible and can be adapted easily to different types of parts [1,11]. Moreover, they are usually more robust and easier to implement. In particular, methods that do not require sensors are favored [1, 3, 7, 9, 11].

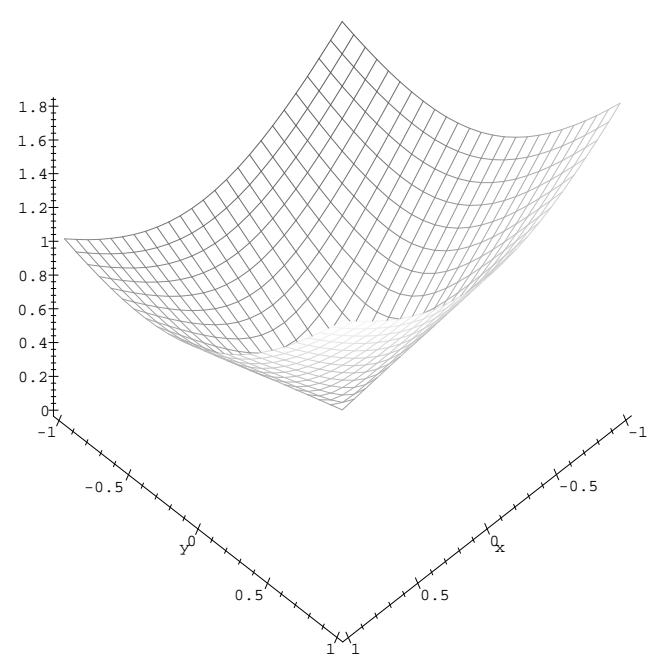

Figure 1: Radial-constant potential field $u(x, y)=\sqrt{x^{2}+y^{2}}+$ $.4 y$. The corresponding force field uniquely positions and orients non-symmetric and some symmetric parts.

One of the alternatives proposed in [11] consists in orienting a polygonal part by a sequence of squeezes performed by two parallel jaw grippers. Given a polygonal part, this work proposed an algorithm that computes the best sequence of squeezes that uniquely orients the convex hull of the part no matter what the initial configuration is.

Another alternative is the use of programmable force fields implemented on a plane [4, 14]. A part lying on the field is subjected to a resultant force and torque that move the part toward a stable equilibrium configuration, if one exists. This technique requires no sensing. Current technology permits the implementation of certain force fields in the microscale with MEMS actuators [4] and in the macroscale using mechanical devices [15], or air jets actuators [2]. Vibrating plates can also be used to produce a force field in the plane [3, 4].

A theory for programmable force fields has been developed in [4] and the reader is referred to this paper for a com- 
prehensive discussion on the work of Böhringer and Donald on the theory and uses of programmable force fields. A number of force fields have been proposed for part manipulation, including the constant, the radial, the inertial field, and their combinations. Strategies that include squeeze fields or a combination of radial and squeeze fields have been developed to position and orient parts. These strategies are based on the sequential application of a number of fields. Their implementation does not require sensing but requires a clock. Complexity (i.e., the number of squeezes necessary to accomplish a task) is an important issue in this context and minimal complexity, which could eliminate the need of a clock, is desirable. Significant progress has been made toward this goal for the task of positioning and orienting a part. An elliptic force field that gives rise to two stable equilibria for non symmetric parts was proposed in [12]. A long standing conjecture of Böhringer and Donald stating that the combination of a unit radial field and a constant field can orient uniquely any non-symmetric part was recently proved in [5]. The field that achieves unique equilibrium arises from a potential; the latter is drawn in Figure 1. The condition for unique orientation under the radial and constant field is the following: under the radial field only and at equilibrium, the center of mass of the part is not above the origin of the field. In this paper, we investigate the case where this condition is not fulfilled and we give an additional condition under which the part has exactly two stable equilibrium configurations. We restrict ourselves to a theoretical investigation of the problem in this paper trying to understand the limitations of programmable force fields.

This paper is organized as follows. Section 2 outlines the conditions for equilibria. Section 3 discusses the properties of the unit radial field. Section 4 presents the radial-constant field and its properties. Our main result is in Section 5. We conclude with a discussion in Section 6.

\section{Conditions for Equilibrium}

Let $\mathbf{f}(x, y)$ be a force field in the plane and let us consider a part occupying a surface $S$ in a reference configuration $\mathbf{q}_{0}$, with center of mass at the origin. Let us denote by $S_{\mathbf{q}}$ the surface occupied by the same part in configuration $\mathbf{q}=(x, y, \theta)$ where $\mathbf{r}_{G}=(x, y)$ is the position of the center of mass $G$ of the part and $\theta$ is the orientation. When placed on a force field, the part is subjected to a resultant force and torque given by:

$$
\begin{aligned}
\mathbf{F}(\mathbf{q}) & =\int_{S \mathrm{q}} f(\mathbf{r}) d \mathbf{r} \\
M(\mathbf{q}) & =\int_{S \mathrm{q}}\left(\mathbf{r}-\mathbf{r}_{G}\right) \times f(\mathbf{r}) d \mathbf{r} .
\end{aligned}
$$

An equilibrium configuration is a configuration where the force and torque vanish.
Potential fields As described in [4], if $\mathbf{f}(x, y)$ derives from a potential field, we can define a potential field in the configuration space by integration over the surface occupied by the part. This field is called the lifted potential field and is defined as follows:

$$
V(\mathbf{q})=\int_{S \mathrm{q}} v(\mathbf{r}) d \mathbf{r}
$$

The stable equilibrium configurations of a part subjected to a potential force field are the local minima of the lifted potential field. In this paper, we are going to use this property since the fields we use derive from potential functions.

\section{Unit Radial Field}

The unit radial field is defined by $\mathbf{f}(x, y)=$ $-\frac{1}{\sqrt{x^{2}+y^{2}}}(x, y)$ and derives from the potential function

$$
v(x, y)=\sqrt{x^{2}+y^{2}}
$$

over the plane. This field is combined with a constant field to achieve unique orientation. We first examine the properties of the radial field.

\subsection{The Lifted Potential Field}

The unit radial field is symmetric by rotation about the origin. For this reason, if we rotate the part about the origin from a given configuration, the lifted potential field $V$ remains constant. Exploiting this idea, we define a new system of coordinates over the configuration space by:

$$
\begin{aligned}
& X=\cos \theta x+\sin \theta y, \\
& Y=-\sin \theta x+\cos \theta y .
\end{aligned}
$$

Two configurations obtained from one another by a rotation about the origin have the same $(X, Y)$ and therefore, expressed in the $(X, Y, \theta)$ system of coordinates, the lifted radial potential field depends only on $(X, Y)$ and can be written:

$$
V(X, Y)=\int_{S} v(X+\xi, Y+\eta) d \xi d \eta
$$

The key property of our reasoning is that $V(X, Y)$ as a function of $(X, Y)$ has a unique local minimum [5].

\subsection{Unique Minimum of $V(X, Y)$}

We will need the following proposition. Note that our discussion below provides an alternative proof to [4] for the existence of the unique minimum. The proof below was also given in [5] but we repeat it here for completeness. 


\section{Proposition 1}

(i) The Hessian of $V(X, Y)$ is positive definite
everywhere
(ii) $V(X, Y)$ has a unique local minimum $\left(X_{0}, Y_{0}\right)$

Proof: (i) Using common results from Lebesgue theory of integration, it can be established that $V(X, Y)$ is $C^{3}$. The Hessian of $V(X, Y)$ can be written

$$
\text { Hess } V(X, Y)=\left(\begin{array}{ll}
\frac{\partial^{2} V}{\partial X^{2}}(X, Y) & \frac{\partial^{2} V}{\partial X}(X, Y) \\
\frac{\partial^{2} V}{\partial X \partial Y}(X, Y) & \frac{\partial^{2} V}{\partial Y^{2}}(X, Y)
\end{array}\right)
$$

where the partial derivatives of $V(X, Y)$ are obtained by differentiating $v(X+\xi, Y+\eta)$ in the integral in equation (2), using expression (1). Hess $V(X, Y)$ is a symmetric matrix and therefore is diagonalizable. The Hessian of $V(X, Y)$ is positive definite iff its eigenvalues are both positive. The sum and product of these eigenvalues are respectively the trace and determinant of Hess $V(X, Y)$. Therefore, Hess $V(X, Y)$ is positive definite iff its determinant and trace are both positive. Let us compute the second partial derivatives of $U_{\theta, \delta}$ :

$$
\begin{aligned}
\frac{\partial^{2} U_{\theta, \delta}}{\partial X^{2}} & =\int_{S} \frac{(Y+\eta)^{2}}{\left((X+\xi)^{2}+(Y+\eta)^{2}\right)^{3 / 2}} d \xi d \eta \\
\frac{\partial^{2} U_{\theta, \delta}}{\partial Y^{2}} & =\int_{S} \frac{(X+\xi)^{2}}{\left((X+\xi)^{2}+(Y+\eta)^{2}\right)^{3 / 2}} d \xi d \eta \\
\frac{\partial^{2} U_{\theta, \delta}}{\partial X \partial Y} & =\int_{S}-\frac{(X+\xi)(Y+\eta)}{\left((X+\xi)^{2}+(Y+\eta)^{2}\right)^{3 / 2}} d \xi d \eta
\end{aligned}
$$

From these expressions, we deduce easily that $\operatorname{tr}$ Hess $U_{\theta, \delta}(X, Y)>0$. Then using the identities $\left(\int f(\xi) d \xi\right)\left(\int g(\xi) d \xi\right)=\iint f(\xi) g(\eta) d \xi d \eta=$ $\iint f(\eta) g(\xi) d \xi d \eta$, we have:

$$
\begin{aligned}
& \operatorname{det} \text { Hess } U_{\theta, \delta}=\frac{\partial^{2} U_{\theta, \delta}}{\partial X^{2}} \frac{\partial^{2} U_{\theta, \delta}}{\partial Y^{2}}-\left(\frac{\partial^{2} U_{\theta, \delta}}{\partial X \partial Y}\right)^{2} \\
& =\iint \frac{Y_{1}^{2} X_{2}^{2}-X_{1} Y_{1} X_{2} Y_{2}}{\left(X_{1}^{2}+Y_{1}^{2}\right)^{3 / 2}\left(X_{2}^{2}+Y_{2}^{2}\right)^{3 / 2}} \\
& =\frac{1}{2} \iint \frac{Y_{1}^{2} X_{2}^{2}+Y_{2}^{2} X_{1}^{2}}{\left(X_{1}^{2}+Y_{1}^{2}\right)^{3 / 2}\left(X_{2}^{2}+Y_{2}^{2}\right)^{3 / 2}} \\
& +\frac{1}{2} \iint \frac{-2 X_{1} Y_{1} X_{2} Y_{2}}{\left(X_{1}^{2}+Y_{1}^{2}\right)^{3 / 2}\left(X_{2}^{2}+Y_{2}^{2}\right)^{3 / 2}} \\
& =\frac{1}{2} \iint \frac{\left(Y_{1} X_{1}-Y_{2} X_{1}\right)^{2}}{\left(X_{1}^{2}+Y_{1}^{2}\right)^{3 / 2}\left(X_{2}^{2}+Y_{2}^{2}\right)^{3 / 2}}>0 .
\end{aligned}
$$

where $X_{1}=X+\xi_{1}, X_{2}=X+\xi_{2}, Y_{1}=Y+\eta_{1}$, $Y_{2}=Y+\eta_{2}$ and $d \xi_{1} d \eta_{1} d \xi_{2} d \eta_{2}$ has been omitted to make the notation clearer.

(ii) $V(X, Y)$ is convex. As $v(x, y)$ tends toward infinity with $(x, y)$, so does $V(X, Y)$ therefore, $V(X, Y)$ has one and only one local minimum.

Pivot point According to Proposition 1, the set of equilibrium configurations of a part subjected to a unit radial field

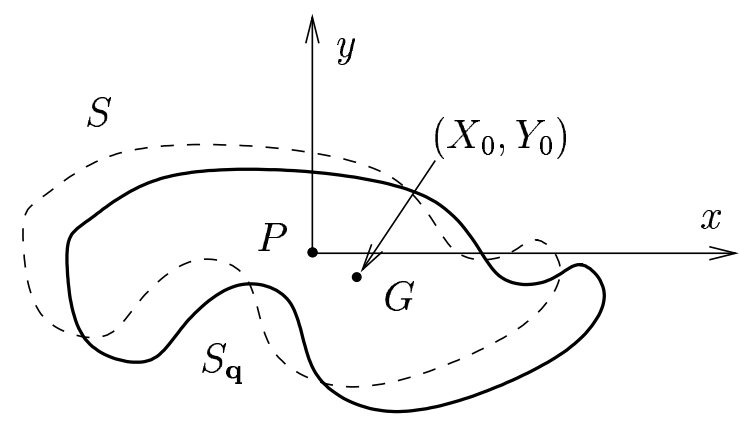

Figure 2: In the equilibrium configuration corresponding to $\theta=$ 0 , the center of mass $G$ is at $\left(X_{0}, Y_{0}\right)$.

is stable by rotation of the part about the origin of the field. For all these configurations, the point of the part situated at the origin is called the pivot point of the part and is denoted by $P$ (see Figure 2 ).

\section{Radial-Constant Field}

We now add to the unit radial field, a constant field of magnitude $\delta$ oriented toward the negative direction of the $y$-axis. This force field derives from the potential $\delta y$. Figure 1 shows the corresponding potential function. The lifted potential field of this combination is expressed as follows in the $(X, Y, \theta)$ system of coordinates

$$
U_{\delta}(X, Y, \theta)=V(X, Y)+\delta|S|(\sin \theta X+\cos \theta Y),
$$

where $|S|$ is the area of $S$. For clarity purposes, we define the following functions

$$
U_{\theta, \delta}(X, Y)=U(X, Y, \theta, \delta)=U_{\delta}(X, Y, \theta),
$$

where the variables we put in the subscript are considered constant.

\subsection{Equilibrium curve}

The second term of the right hand side of expression (3) is linear in $(X, Y)$. For this reason, $U_{\theta, \delta}$ has the same second order partial derivatives as $V$ and Hess $U_{\theta, \delta}=$ Hess $V$ is positive definite (Proposition 1). For any fixed value of $\theta$ and $\delta<1, U_{\theta, \delta}(X, Y)$ tends toward infinity with $(X, Y)$. Thus, $U_{\theta, \delta}$ has a unique local minimum. We denote by $\left(X^{*}(\theta, \delta), Y^{*}(\theta, \delta)\right)$ this local minimum that we can express in the standard system of coordinates by

$$
\begin{aligned}
& x^{*}(\theta, \delta)=\cos \theta X^{*}(\theta, \delta)-\sin \theta Y^{*}(\theta, \delta), \\
& y^{*}(\theta, \delta)=\sin \theta X^{*}(\theta, \delta)+\cos \theta Y^{*}(\theta, \delta) .
\end{aligned}
$$

For each value of $\delta<1$, these local minima define a curve of parameter $\theta$ that we call equilibrium curve [5].

If we define

$$
F(X, Y, \theta, \delta)=\left(\begin{array}{c}
\frac{\partial U}{\partial X}(X, Y, \theta, \delta) \\
\frac{\partial U}{\partial Y}(X, Y, \theta, \delta)
\end{array}\right)
$$


$\left(X^{*}, Y^{*}\right)$ is implicitly defined by: $F\left(X^{*}, Y^{*}, \theta, \delta\right)=0$. For each $\theta$ and $\delta$ fixed, the partial function $F_{\theta, \delta}(X, Y)$ is $C^{2}$ and its differential is exactly the Hessian of $V(X, Y)$. This Hessian is invertible everywhere according to Proposition 1. Therefore, the implicit function theorem enables us to state that $X^{*}, Y^{*}$ and thus $x^{*}, y^{*}$ are $C^{2}$ functions of $\theta$ and $\delta$.

\subsection{Condition for Local Minima of $U_{\delta}$}

As explained above, we are interested in the local minima of the lifted potential field induced by the combination of a unit radial field and a constant field. These local minima are the stable equilibrium configurations of the part subjected to this field. A local minimum of $U_{\delta}$ is necessarily on the equilibrium curve. To find these local minima, we just have to look at the lifted potential along the equilibrium curve. Our minimization problem thus becomes onedimensional. Let us define

$$
U_{\delta}^{*}(\theta)=U\left(X^{*}(\theta, \delta), Y^{*}(\theta, \delta), \theta, \delta\right),
$$

be the value of the lifted potential along the equilibrium curve and let us compute the derivative of this function w.r.t. $\theta$ :

$$
\begin{aligned}
\frac{d U_{\delta}^{*}}{d \theta}(\theta) & =\frac{\partial U}{\partial X}\left(X^{*}(\theta, \delta), Y^{*}(\theta, \delta), \theta, \delta\right) \frac{\partial X^{*}}{\partial \theta}(\theta, \delta) \\
& +\frac{\partial U}{\partial Y}\left(X^{*}(\theta, \delta), Y^{*}(\theta, \delta), \theta, \delta\right) \frac{\partial Y^{*}}{\partial \theta}(\theta, \delta) \\
& +\frac{\partial U}{\partial \theta}\left(X^{*}(\theta, \delta), Y^{*}(\theta, \delta), \theta, \delta\right) .
\end{aligned}
$$

As $X^{*}$ and $Y^{*}$ minimize $U$ in $(X, Y)$, the two first terms of the right hand side of the above equation are zero. The third term can be computed from (3). We get

$$
\begin{aligned}
\frac{d U_{\delta}^{*}}{d \theta}(\theta) & =\delta|S|\left(\cos \theta X^{*}(\theta, \delta)-\sin \theta Y^{*}(\theta, \delta)\right) \\
& =\delta|S| x^{*}(\theta, \delta) .
\end{aligned}
$$

This equation means that the variation of the lifted potential along the equilibrium curve depends on the abscissa of the translational minimum. This property leads directly to the following proposition.

Proposition 2 The stable equilibrium configurations of a part placed on the combination of a radial and a constant field are, given by the values of $\theta$ where the equilibrium curve crosses the $y$-axis from left to right when $\theta$ increases.

In the general case, we do not have an exact expression of $x^{*}(\theta, \delta)$ to apply Proposition 2. However, for small values of $\delta$, it is possible to guess the shape of the equilibrium curve from the values of $x^{*}(\theta, 0)$ and $\frac{\partial x^{*}}{\partial \delta}(\theta, 0)$ using Taylor expansion:

$$
x^{*}(\theta, \delta)=x^{*}(\theta, 0)+\delta \frac{\partial x^{*}}{\partial \delta}(\theta, 0)+o(\delta) .
$$

This is the topic of the next section.

\section{Unique and Non-Unique Orientation}

Let us recall that $\left(X^{*}(\theta, 0), Y^{*}(\theta, 0)\right)=\left(X_{0}, Y_{0}\right)$ is the minimum of $V(X, Y)$ and thus does not depend on $\theta$. Using (4),

$$
x^{*}(\theta, 0)=\cos \theta X_{0}-\sin \theta Y_{0} .
$$

\subsection{Unique Stable Equilibrium}

If $\left(X_{0}, Y_{0}\right) \neq(0,0)$, i.e. the pivot point and the center of mass are distinct, the equilibrium curve for $\delta=0$ is a circle centered at the origin. This circle crosses only once the $y$ axis from left to right. In [5], we proved that by continuity of $x^{*}$ and its partial derivative $\frac{\partial x^{*}}{\partial \theta}$, this property remained true for small values of $\delta$ and therefore that the part had a unique equilibrium configuration.

We are now interested in the case $\left(X_{0}, Y_{0}\right)=(0,0)$, where the pivot point and center of mass are the same. This condition arises for symmetric parts like rectangle or regular polygons.

\subsection{Two Stable Equilibria}

When $\left(X_{0}, Y_{0}\right)=(0,0), x^{*}(\theta, 0)$ is uniformly zero and cannot be used as an approximation of $x^{*}(\theta, \delta)$ for small $\delta$. To predict the sign of $x^{*}(\theta, \delta)$ for small $\delta$, we use formula (6) that becomes:

$$
x^{*}(\theta, \delta)=\delta \frac{\partial x^{*}}{\partial \delta}(\theta, 0)+o(\delta) .
$$

In the rest of this section, we will determine an expression of $\frac{\partial x^{*}}{\partial \delta}(\theta, 0)$ and we will show that for small $\delta$, the values of $\theta$ where $x^{*}(\theta, \delta)$ and $\frac{\partial x^{*}}{\partial \delta}(\theta, 0)$ vanish are close to each other.

Computation of $\frac{\partial x^{*}}{\partial \delta}(\theta, 0)$. Let us differentiate (4) w.r.t. $\delta$, we get

$$
\frac{\partial x^{*}}{\partial \delta}(\theta, 0)=\cos \theta \frac{\partial X^{*}}{\partial \delta}(\theta, 0)-\sin \theta \frac{\partial Y^{*}}{\partial \delta}(\theta, 0) .
$$

$\frac{\partial x^{*}}{\partial \delta}(\theta, 0)$ can be deduced from $\frac{\partial X^{*}}{\partial \delta}(\theta, 0)$ and $\frac{\partial Y^{*}}{\partial \delta}(\theta, 0)$. Let us recall that $\left(X^{*}, Y^{*}\right)$ minimizes $U$ in $(X, Y)$ and therefore that

$$
\begin{aligned}
& \frac{\partial U_{\delta}}{\partial X}\left(X^{*}(\theta, \delta), X^{*}(\theta, \delta), \theta\right)=0 \\
& \frac{\partial U_{\delta}}{\partial Y}\left(X^{*}(\theta, \delta), X^{*}(\theta, \delta), \theta\right)=0
\end{aligned}
$$

Replacing $U_{\delta}$ by expression (3) we get:

$$
\begin{aligned}
& \frac{\partial V}{\partial X}\left(X^{*}(\theta, \delta), Y^{*}(\theta, \delta)\right)+\delta|S| \sin \theta=0, \\
& \frac{\partial V}{\partial Y}\left(X^{*}(\theta, \delta), Y^{*}(\theta, \delta)\right)+\delta|S| \cos \theta=0 .
\end{aligned}
$$


Differentiating these equations w.r.t. $\delta$, we have

$$
\begin{array}{r}
\frac{\partial^{2} V}{\partial X^{2}}\left(X^{*}, Y^{*}\right) \frac{\partial X^{*}}{\partial \delta}+\frac{\partial^{2} V}{\partial X \partial Y}\left(X^{*}, Y^{*}\right) \frac{\partial Y^{*}}{\partial \delta} \\
+|S| \sin \theta=0 \\
\frac{\partial^{2} V}{\partial X \partial Y}\left(X^{*}, Y^{*}\right) \frac{\partial X^{*}}{\partial \delta}+\frac{\partial^{2} V}{\partial Y^{2}}\left(X^{*}, Y^{*}\right) \frac{\partial Y^{*}}{\partial \delta} \\
+|S| \cos \theta=0,
\end{array}
$$

where $X^{*}$ and $Y^{*}$ are evaluated at $(\theta, \delta)$. Now, taking $\delta=0$ in these expressions, we get

$$
\text { Hess } V(0,0)\left(\begin{array}{c}
\frac{\partial X^{*}}{\partial \delta}(\theta, 0) \\
\frac{\partial Y^{*}}{\partial \delta}(\theta, 0)
\end{array}\right)+|S|\left(\begin{array}{c}
\sin \theta \\
\cos \theta
\end{array}\right)=0 \text {. }
$$

This is a linear system of equations whose coefficients are the second order partial derivatives of $V$ at $(0,0)$. Let us recall that the Hessian of $V$ is symmetric positive definite everywhere. Thus it is diagonalizable, i.e. there exist two positive number $1 / \lambda_{1}$ and $1 / \lambda_{2}$, and a rotation matrix $R_{\psi}$ such that

$$
\text { Hess } V(0,0)=R_{-\psi}\left(\begin{array}{ll}
\frac{1}{\lambda_{1}} & 0 \\
0 & \frac{1}{\lambda_{2}}
\end{array}\right) R_{\psi} .
$$

Multiplying (8) by Hess $V(0,0)^{-1}$, we get:

$$
\left(\begin{array}{c}
\frac{\partial X^{*}}{\partial \delta^{*}}(\theta, 0) \\
\frac{\partial Y^{*}}{\partial \delta}(\theta, 0)
\end{array}\right)=R_{-\psi}\left(\begin{array}{cc}
\lambda_{1} & 0 \\
0 & \lambda_{2}
\end{array}\right) R_{\psi}\left(\begin{array}{c}
-|S| \sin \theta \\
-|S| \cos \theta
\end{array}\right)
$$

and using (7),

$$
\frac{\partial x^{*}}{\partial \delta}(\theta, 0)=\frac{1}{2}|S|\left(\lambda_{2}-\lambda_{1}\right) \sin (2(\theta+\psi)) .
$$

The following proposition states that for small $\delta, x^{*}(\theta, \delta)$ vanishes close to the values of $\theta$ where $\frac{\partial x^{*}}{\partial \delta}(\theta, 0)$ vanishes.

Proposition 3 If the pivot point $P$ and the center of mass $G$ are the same and if Hess $V(0,0)$ has two different eigenvalues, then the part has two stable equilibrium configurations for small $\delta$. In these configurations, the axis of the part coinciding in configuration $\mathbf{q}_{0}$ with $(O x)$ aligns with the eigenvector of Hess $V(0,0)$ of larger eigenvalue.

Proof: Without loss of generality, we assume that $\frac{1}{\lambda_{2}}$ is the larger eigenvalue: $\lambda_{1}-\lambda_{2}>0$. The idea of the proof is that as $x^{*}(\theta, 0)=0$, the sign of $x^{*}(\theta, \delta)$ for small $\delta$ is given by the sign of $\frac{\partial x^{*}}{\partial \delta}(\theta, 0)$. As this latter function vanishes 4 times ( 2 times from $x<0$ to $x>0$ ) when $\theta$ describes the unit circle, so does $x^{*}(\theta, \delta)$ and the part has two stable equilibrium configurations.

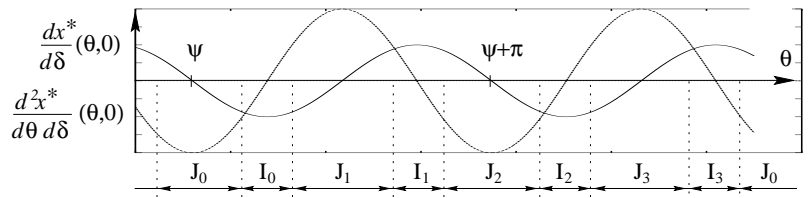

Figure 3: Partial derivatives of the equilibrium curve.
As we consider only small values of $\delta$, we can constrain $(\theta, \delta)$ to remain on a compact set, so that $x^{*}$ and its derivatives are uniformly continuous. We now divide the unit circle into the following intervals:

$$
\begin{aligned}
& I_{k}=\left[-\psi+\frac{\pi}{6}+\frac{k \pi}{2},-\psi+\frac{(k+1) \pi}{2}-\frac{\pi}{6}\right] \\
& J_{k}=\left[-\psi-\frac{\pi}{6}+\frac{k \pi}{2},-\psi+\frac{\pi}{6}+\frac{k \pi}{2}\right]
\end{aligned}, k \in\{0,1,2,3\},
$$

as shown in Figure 3. The first step is to show that for $\delta$ small enough, $x^{*}(\theta, \delta)$ does not vanish over the $I_{k}$. We notice that

$$
\begin{array}{ll}
\forall \theta \in I_{0} \cup I_{2}, & \frac{\partial x^{*}}{\partial \delta}(\theta, 0)<-|S|\left(\lambda_{1}-\lambda_{2}\right) \frac{\sqrt{3}}{4}, \\
\forall \theta \in I_{1} \cup I_{3}, & \frac{\partial x^{*}}{\partial \delta}(\theta, 0)>|S|\left(\lambda_{1}-\lambda_{2}\right) \frac{\sqrt{3}}{4} .
\end{array}
$$

From the uniform continuity of $\frac{\partial x^{*}}{\partial \delta}$, there exists $\delta_{1}>0$ such that:

$$
\forall \delta \in\left[0, \delta_{1}\right], \begin{array}{ll}
\forall \theta \in I_{0} \cup I_{2}, & \frac{\partial x^{*}}{\partial \delta^{*}}(\theta, \delta)<0 \\
\forall \theta \in I_{1} \cup I_{3}, & \frac{\partial x^{*}}{\partial \delta}(\theta, \delta)>0
\end{array}
$$

From these inequalities, and since $x^{*}(\theta, 0)=0$, we can deduce that for any $\delta<\delta_{1}, x^{*}(\theta, \delta)$ is positive over $I_{1}$ and $I_{3}$ and negative over $I_{0}$ and $I_{2}$.

The second step is to show that $x^{*}(\theta, \delta)$ vanishes only once over each of the $J_{k}$. We can differentiate (9) with respect to $\theta$ to get

$$
\frac{\partial^{2} x^{*}}{\partial \theta \partial \delta}(\theta, 0)=-|S|\left(\lambda_{1}-\lambda_{2}\right) \cos (2(\theta-\psi)) .
$$

Over the $J_{k}$, the partial derivative $\frac{\partial^{2} x^{*}}{\partial \theta \partial \delta}$ is lower bounded in absolute value:

$$
\begin{array}{ll}
\forall \theta \in J_{0} \cup J_{2}, & \frac{\partial^{2} x^{*}}{\partial \theta \partial \delta}(\theta, 0)<-\frac{|S|}{2}\left(\lambda_{1}-\lambda_{2}\right), \\
\forall \theta \in J_{1} \cup J_{3}, & \frac{\partial^{2} x^{*}}{\partial \theta \partial \delta}(\theta, 0)>\frac{|S|}{2}\left(\lambda_{1}-\lambda_{2}\right) .
\end{array}
$$

Using the uniform continuity of $\frac{\partial^{2} x^{*}}{\partial \theta \partial \delta}$, we can deduce that there exists $\delta_{5}>0$ such that

$$
\forall \delta \in\left[0, \delta_{2}\right], \begin{array}{ll}
\forall \theta \in J_{0} \cup J_{2}, & \frac{\partial^{2} x^{*}}{\partial \theta \partial \delta}(\theta, \delta)<0, \\
\forall \theta \in J_{1} \cup J_{3}, & \frac{\partial^{2} x^{*}}{\partial \theta \partial \delta}(\theta, \delta)>0,
\end{array}
$$

and as $\frac{\partial x^{*}}{\partial \theta}(\theta, 0)=0$, the above inequalities lead to:

$$
\forall \delta \in\left[0, \delta_{2}\right], \begin{array}{ll}
\forall \theta \in J_{0} \cup J_{2}, & \frac{\partial x^{*}}{\partial \theta_{*}}(\theta, \delta)<0, \\
\forall \theta \in J_{1} \cup J_{3}, & \frac{\partial x^{*}}{\partial \theta}(\theta, \delta)>0 .
\end{array}
$$

As $\frac{\partial x^{*}}{\partial \theta}(\theta, \delta)$ keeps a constant sign over each interval $J_{k}$, $x^{*}(\theta, \delta)$ can vanish at most once on each of them. As $x^{*}$ changes sign between the intervals $I_{k}$, for $\delta<\delta_{1}$, it changes sign exactly once between these intervals defining thus two stable and two unstable equilibrium configurations for the part.

Notice that in this proof, the intervals $J_{k}$ can be chosen as small as desired around the values $-\psi+k \frac{\pi}{2}, k \in$ 


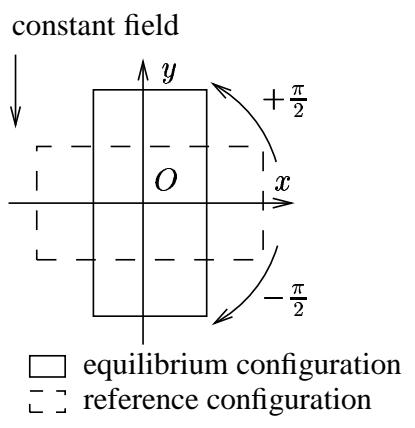

Figure 4: A rectangle in the radial constant field rotates to align its long edges with the constant component of the field.

$\{0,1,2,3\}$, where $\frac{\partial x^{*}}{\partial \delta}(\theta, 0)$ vanishes. This just makes $\delta_{1}$ smaller. Thus, when $\delta$ tends toward 0 , the roots of $x^{*}(\theta, \delta)$ tend toward $-\psi+k \frac{\pi}{2}$. This ensures the second part of the proposition.

Example Let us consider the rectangle defined by the following domain $S=[-2,2] \times[-1,1]$ shown in Figure 4 . Because of the symmetry, both the center of mass and the pivot points are located at the center of the rectangle. When this part is placed in the radial field, we have

$$
\begin{aligned}
& \frac{\partial^{2} V}{\partial X^{2}}(0,0)=8 \operatorname{argsinh} \frac{1}{2} \approx 3.85, \\
& \frac{\partial^{2} V}{\partial X \partial Y}(0,0)=0, \\
& \frac{\partial^{2} V}{\partial Y^{2}}(0,0)=4 \operatorname{argsinh} 2 \approx 5.77 .
\end{aligned}
$$

The Hessian is already in diagonal form. The larger eigenvalue is $\frac{\partial^{2} V}{\partial Y^{2}}(0,0)$ and the associated eigenvectors are along the $y$-axis. Thus, for small $\delta$, the main axis of the rectangle aligns with the $y$-axis.

Notice that the rectangle is uniquely positioned up to symmetry under the radial-constant field. Of course, there might exist non geometrically symmetric parts with the same center of mass and pivot point. These parts cannot be positioned uniquely by the radial-constant field.

\section{Discussion and Future Work}

In this paper, we have extended former results on part orienting using a combination of a unit radial field and a small constant field. We proved that some symmetric part can be oriented up to symmetry. In another paper [13], we investigate parts with more symmetries. In the future we would like to investigate if there are force fields other than the ones presented in this paper that can achieve unique positioning and orientation of parts.

Acknowledgments Lydia Kavraki and Florent Lamiraux are partially supported by NSF CAREER Award IRI-970228. In their work on part manipulation with programmable vector fields the authors have benefited greatly from discussions with Karl Böhringer, Bruce Donald, Leo Guibas, and Jean-Claude Latombe.
The authors would also like to thank the members of the Physical Computing group at Rice for their comments.

\section{References}

[1] S. Akella, W. Huang, K. Lynch, and M. Mason. Planar manipulation on a conveyor with a one joint robot. In Giralt and Hirzinger [10], pages 265-276.

[2] D. Biegelsen, W. Jackson, A. Berlin, and P. Cheung. Air jet arrays for precision positional control of flexible media. In Proc. Int. Conf. on Micromechatronics for Information and Precision Equipment, pages 631-634, Tokyo, Japan, 1997.

[3] K.-F. Böhringer, V. Bhatt, and K. Goldberg. Sensorless manipulation using transverse vibrations of a plate. In Proc. IEEE Int. Conf. on Rob. and Autom., pages 1989-1996, 1995.

[4] K.-F. Böhringer, B. Donald, and N. MacDonald. Programmable vector fields for distributed manipulation, with application to mems actuator arrays and vibratory part feeders. Int. J. of Robotics Research, 18:168-200, Feb. 1999.

[5] K.-F. Böhringer, B. R. Donald, L. E. Kavraki, and F. Lamiraux. Part orientation with one or two stable equilibria using programmable vector fields. To appear in the IEEE Transactions on Robotics and Automation.

[6] K.-F. Böhringer, B. R. Donald, and N. C. MacDonald. Upper and lower bounds for programmable vector fields with applications to MEMS and vibratory plate parts feeders. in Algorithms for Robotic Motion and Manipulation, Jean-Paul Laumond and Mark Overmars (Eds), pages 255-276, A. K. Peters, Ltd, 1997.

[7] J. Canny and K. Y. Goldberg. Risc for industrial robotics: recent results and open problems. In Proc. IEEE Int. Conf. on Rob. and Autom., pages 1951-1958, 1994.

[8] M. Erdmann. An exploration of nonprehensile two-palm manipulation: Planning and execution. In Giralt and Hirzinger [10], pages 16-27.

[9] M. Erdmann and M. Mason. An exploration of sensorless manipulation. IEEE Tr. on Rob. and Autom., 4(4):369-379, 1988.

[10] G. Giralt and G. Hirzinger, editors. Robotics Research Springer, 1996.

[11] K. Y. Goldberg. Orienting polygonal parts without sensors. Algorithmica, 10:201-225, 1993.

[12] L. E. Kavraki. Part orientation with programmable vector fields: Two stable equilibria for most parts. In Proc. IEEE Int. Conf. on Robotics and Automation, pages 2446-2451, 1997.

[13] F. Lamiraux and L. E. Kavraki. Positioning Symmetric and Non-Symmetric Parts using Radial and Constant Force Fields To appear in the Workshop on the Algorithmic Foundations of Robotics, 2000.

[14] M. Coutinho and P. Will. The intelligent motion surface: a hardware/software tool for the assembly of meso-scale devices. In IEEE Int. Conf. on Robotics and Automation (ICRA), Albuquerque, NM, 1997.

[15] J. Luntz, W. Messner, and H. Choset. Velocity field design for parcel manipulation on the virtual vehicule, a discrete distributed actuator array. In P. Agarwal, L. E. Kavraki, and M. Mason, editors, Robotics: The Algorithmic Perspective, pages 35-47. AK Peters, Natick, MA, 1998.

[16] J. Wiegley, K. Goldberg, M. Peshkin, and M. Brokowski. A complete algorithm for designing passive fences to orient parts. In Proc. Int. Conf. on Rob. and Autom., pages 11331139, 1996. 